\title{
Association between Smoking and the Progression of Computed Tomography Findings in Chronic Pancreatitis
}

Jeong Woo Lee, Ho Gak Kim, Dong Wook Lee, Jimin Han, Hyuk Yong Kwon, Chang Jin Seo, Ji Hye Oh, Joo Hyoung Lee, Jin Tae Jung, Joong Goo Kwon, and Eun Young Kim

Department of Internal Medicine, Catholic University of Daegu School of Medicine, Daegu, Korea

Background/Aims: Smoking and alcohol intake are two wellknown risk factors for chronic pancreatitis. However, there are few studies examining the association between smoking and changes in computed tomography (CT) findings in chronic pancreatitis. The authors evaluated associations between smoking, drinking and the progression of calcification on CT in chronic pancreatitis. Methods: In this retrospective study, 59 patients with chronic pancreatitis who had undergone initial and follow-up CT between January 2002 and September 2010 were included. Progression of calcification among CT findings was compared according to the amount of alcohol intake and smoking. Results: The median duration of followup was 51.6 months (range, 17.1 to 112.7 months). At initial CT findings, there was pancreatic calcification in 35 patients (59.3\%). In the follow-up CT, progression of calcification was observed in 37 patients (62.7\%). Progression of calcification was more common in smokers according to the multivariate analysis (odds ratio [OR], 9.987; $p=0.006$ ). The amount of smoking was a significant predictor for progression of calcification in the multivariate analysis (OR, 6.051 in less than 1 pack per day smokers; OR, 36.562 in more than 1 pack per day smokers; $p=0.008)$. Conclusions: Continued smoking accelerates pancreatic calcification, and the amount of smoking is associated with the progression of calcification in chronic pancreatitis. (Gut Liver 2016;10:464-469)

Key Words: Pancreatitis, chronic; Calcification; Smoking; Computed tomography

\section{INTRODUCTION}

Chronic pancreatitis is characterized by progressive and irreversible loss of pancreatic structure. The single most important risk factor of chronic pancreatitis is alcohol. ${ }^{1}$ Smoking has been described as an additional risk factor of chronic pancreatitis. ${ }^{2}$ According to recent studies, cigarette smoking is an independent risk factor of chronic pancreatitis and accelerates the progression of pancreatic calcification and functional impairment, ${ }^{3-6}$ and cigarette smoking enhances ethanol-induced pancreatic injury. ${ }^{7}$

The morphologic changes of chronic pancreatitis include calcification of the pancreas, stenosis and dilation of the pancreatic duct, ductal calculus, and parenchymal atrophy. The evaluation of such morphological changes can be made by abdominal ultrasound, computed tomography (CT), endoscopic retrograde cholangiopancreatography (ERCP), and endoscopic ultrasonography. CT provides the most accurate evidence of morphological changes of chronic pancreatitis, which include calcification, pancreatic duct dilatation and stenosis, and pancreatic parenchymal atrophy.

However, there are only few studies about association between smoking and the changes of CT findings. We evaluated association between ongoing cigarette smoking and the changes of CT findings in chronic pancreatitis during midterm followup.

\section{MATERIALS AND METHODS}

\section{Enrollment of patients and data collection}

In this retrospective study, 59 patients with chronic pancreatitis who had undergone initial and follow-up CT scans between January 2002 and September 2010 were included at Daegu Catholic University Medical Center, Daegu, Korea. Data about alcohol consumption and smoking status were collected from the medical record and phone call; data about CT findings of chronic pancreatitis were obtained from archives of abdominal

Correspondence to: Ho Gak Kim

Department of Internal Medicine, Catholic University of Daegu School of Medicine, 33 Duryugongwon-ro 17-gil, Nam-gu, Daegu 42472, Korea

Tel: +82-53-650-4041, Fax: +82-53-624-3281, E-mail: hgkim@cu.ac.kr

Received on July 29, 2014. Revised on May 27, 2015. Accepted on June 23, 2015. Published online November 27,2015

pISSN 1976-2283 eISSN 2005-1212 http://dx.doi.org/10.5009/gnl14289

(a) This is an Open Access article distributed under the terms of the Creative Commons Attribution Non-Commercial License (http://creativecommons.org/licenses/by-nc/4.0) which permits unrestricted non-commercial use, distribution, and reproduction in any medium, provided the original work is properly cited. 
CT. This single center study was approved by our institutional review board as a medical records review.

\section{Investigation of alcohol consumption and smoking status of patients}

Amount of alcohol consumption and smoking status were obtained at the time of initial CT and follow-up CT. One drink of alcohol is 9 to $10 \mathrm{~g}$ of alcohol independent to the kind of liquors in general. The amount of alcohol consumption was graded into low to moderate drinker $(<350 \mathrm{~g} / \mathrm{wk})$ and heavy drinker $(\geq 350$ $\mathrm{g} / \mathrm{wk}$ ). We defined continued drinking as the drinker continuing to drink between initial CT and follow-up CT.

Smoking status was graded into nonsmoker and smoker for more than 1 year when chronic pancreatitis was confirmed with initial CT. When follow-up CT was performed, smoking status was graded into nonsmoker including ex-smoker, smoker of less than 1 pack per day, or smoker of more than 1 pack per day. We defined continued smoking as the smoker continuing to smoke between initial CT and follow-up CT.

\section{Analysis of CT findings of patients}

CT findings of chronic pancreatitis included pancreatic calcification, atrophy of the pancreatic parenchyma, and irregularity of the pancreatic duct. Pancreatic calcification was graded into mild, moderate, and severe using a scoring system, which is composed of distribution of calcification, the ratio of the number of large calcified dots ( $>4 \mathrm{~mm}$ ) and the number of calcified dots. In distribution of calcification, score 1 is when calcification is confined only to the head or body or tail; score 2 is when calcification is confined to two of the head, body or tail; score 3 is when calcification is distributed throughout the entire pancreas. In ratio of large calcified dot, if the ratio is less than one-third, the score is 1 ; If the ratio is from one-third to one-half, the score is 2 ; if the ratio is greater than one-half the score is 3 . In the number of calcified dots, score is 1 when there are less than 10 dots; 2 in 10 to 20 dots; 3 in over 20 dots. The sum of score of calcification was calculated by adding the three scores. The sum of score 1 to 3 was classified as mild, 4 to 6 as moderate, 7 to 9 as severe calcification (Table 1). Progression of calcification was defined as progression of mild calcification into moderate or severe calcification and progression of moder- ate calcification into severe calcification. Parenchymal atrophy and ductal irregularity were identified according to CT finding of pancreatic parenchyma and duct.

\section{Comparison of CT findings between initial CT and follow- up CT}

We investigated the changes of CT findings between initial CT and follow-up CT in the patients with chronic pancreatitis. We evaluated association between alcohol consumption, smoking and the progression of pancreatic calcification among the changes of CT findings. Also, we investigated new events in the patients with chronic pancreatitis during follow-up period such as new-onset diabetes and development of pancreatic cancer.

\section{Statistical analysis}

Statistical analyses were performed using SPSS version 13.0 (SPSS Inc., Chicago, IL, USA). The data were expressed as mean \pm standard deviation for continuous variables and percentage in categorical variables. The independent t-test for continuous variables and chi-square test for categorical variables were used to compare the progression of calcification group and no progression of calcification group. Multivariate logistic regression analysis was used to assess the association between alcohol drinking and smoking and progression of calcification.

Age, sex, alcohol drinking status, smoking status, amount of alcohol consumption, and amount of smoking were included in the multivariate analysis. A p-value less than 0.05 was considered to be statistically significant.

\section{RESULTS}

\section{Baseline characteristics of the patients}

Of the 59 patients, 53 patients (89.8\%) were male and six patients were female. The mean age of the patients was $54.5 \pm 14.7$ years. Forty-seven patients (79.7\%) were smokers and 12 patients (20.3\%) were nonsmokers when chronic pancreatitis was diagnosed by the initial abdominal CT. Fifty patients (84.7\%) were alcohol drinkers and nine patients (15.3\%) were nondrinkers at the time of initial CT. Etiology of chronic pancreatitis was as follows: alcohol in 47 (79.7\%), idiopathic in nine (15.3\%), pancreas divisum in two, and bile duct stone in one. The medi-

Table 1. Grades of Pancreatic Calcification

\begin{tabular}{lccc}
\hline & & Score & 3 \\
\cline { 2 - 4 } & 1 & 2 & Distributed throughout the head, \\
bistribution of calcification & Confined to only one region & Confined to two regions of & and tail \\
hatio of large calcified dot $(>4 \mathrm{~mm})^{*}$ & $<1 / 3$ & $1 / 3-1 / 2$ & $>1 / 2$ \\
No. of calcified dots & $<10$ & $10-20$ & $>20$ \\
\hline
\end{tabular}

Sum of scores 1 to 3 was classified as mild, 4 to 6 as moderate, 7 to 9 as severe.

*The ratio of the number of calcified dots larger than $4 \mathrm{~mm}$ to the total number of calcified dots. 
Table 2. Baseline Characteristics of 59 Patients

\begin{tabular}{lc}
\multicolumn{1}{c}{ Characteristic } & Value \\
\hline Age, yr & $54.5 \pm 14.7$ \\
Sex, male/female & $53 / 6$ \\
Smoking status at the time of initial CT & $47(79.7)$ \\
Smoker, pack-year & $2(3.4)$ \\
$<10$ & $24(40.7)$ \\
10-20 & $21(35.6)$ \\
$>30$ & $12(20.3)$ \\
Nonsmoker & $50(84.7)$ \\
Drinking status at the time of diagnosis & $9(15.3)$ \\
Drinker & \\
Nondrinker & \\
Cause of chronic pancreatitis & $47(79.7)$ \\
Alcohol & $9(15.3)$ \\
Idiopathic & $2(3.4)$ \\
Pancreas divisum & $1.6(17.1-112.7)$ \\
Bile duct stone & \\
Duration of follow-up, mo & \\
\hline
\end{tabular}

Data are presented as mean \pm SD, number (\%), or median (range).

CT, computed tomography.

an duration of follow-up was 51.6 months (range, 17.1 to 112.7 months) (Table 2).

\section{CT findings at initial diagnosis and follow-up of chronic pancreatitis}

According to initial CT, there were calcification in 35 patients (59.3\%), atrophy in 21 patients (35.6\%) and ductal irregularity in 18 patients (30.5\%). Mild calcification of score 1 to 3 was seen in 14 patients (23.7\%), moderate calcification of score 4 to 6 in nine patients (15.3\%) and severe calcification of score 7 to 9 in 12 patients (20.3\%).

According to follow-up CT after the median follow-up of 51.6 months, there were calcification in 52 patients (88.1\%), atrophy in 42 patients (71.2\%), and ductal irregularity in 25 patients (42.4\%). Mild calcification was seen in 13 patients (22.0\%), moderate calcification in 14 patients (23.7\%) and severe calcification in 25 patients (42.4\%) (Fig. 1). In the follow-up CT, progression of calcification was seen in 37 patients (62.7\%) (Table 3).

\section{Relation between the change of CT findings and con- sumption of alcohol during follow-up}

During follow-up, 38 of 59 patients stopped drinking or kept abstinence and 21 patients continued alcohol drinking. On the follow-up CT, progression of calcification was seen in 20 of 38 patients (52.6\%) who stopped drinking or kept abstinence and in 17 of 21 patients (81\%) who continued alcohol drinking. Progression of calcification was more common in patients with con-

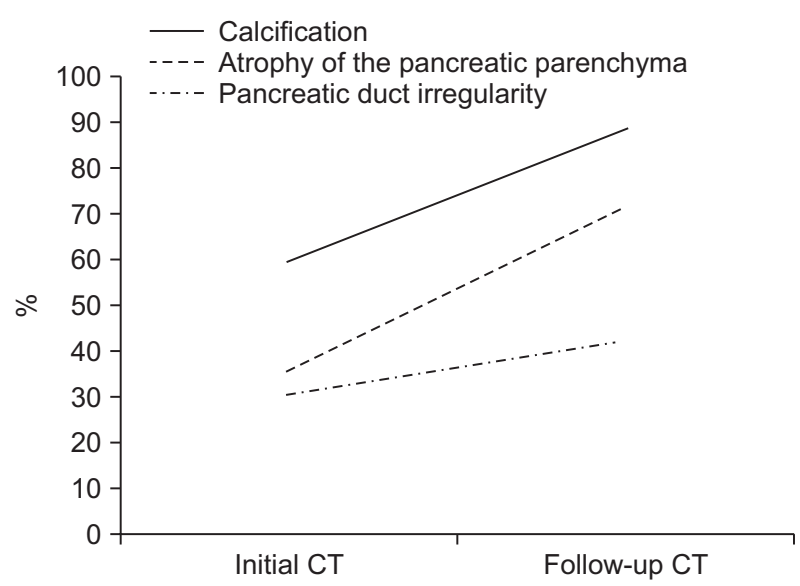

Fig. 1. The change in chronic pancreatitis findings between initial computed tomography (CT) and follow-up CT.

Table 3. Finding of Computed Tomography in 59 Patients with Chronic Pancreatitis

\begin{tabular}{lccc}
\hline & $\begin{array}{c}\text { Initial CT } \\
(\mathrm{n}=59)\end{array}$ & $\begin{array}{c}\text { Follow-up CT } \\
(\mathrm{n}=59)\end{array}$ & $\begin{array}{c}\text { Positive progression } \\
\text { of CT findings }\end{array}$ \\
\hline Calcification & $35(59.3)$ & $52(88.1)$ & $37(62.7)$ \\
Mild & $14(23.7)$ & $13(22.0)$ & \\
Moderate & $9(15.3)$ & $14(23.7)$ & \\
Severe & $12(20.3)$ & $25(42.4)$ & \\
\hline
\end{tabular}

Data are presented as number (\%).

CT, computed tomography.

tinued alcohol drinking than in patients who stopped drinking or kept abstinence in the univariate analysis $(\mathrm{p}=0.048)$ (Table 4) .

In the analysis of amount of alcohol consumption and progression of CT findings during follow-up, the 21 patients who continued alcohol drinking were divided into low to moderate drinker ( $<350 \mathrm{~g} / \mathrm{wk}, \mathrm{n}=14$ ) and heavy drinker ( $\geq 350 \mathrm{~g} / \mathrm{wk}, \mathrm{n}=7$ ). Progression of calcification was not associated with amount of alcohol consumption in univariate analysis $(\mathrm{p}=0.089)$ (Table 4).

\section{Relation between the change of CT findings and status of smoking during follow-up}

During follow-up, the 21 patients stopped smoking or kept nonsmoking and 38 patients continued smoking. On the followup CT, there was progression of calcification in seven of 21 patients (33.3\%) who stopped smoking or kept nonsmoking and in 30 of 38 patients (78.9\%) who continued smoking. Progression of calcification was more common in patients who continued smoking than in patients who stopped smoking or kept nonsmoking in univariate analysis ( $\mathrm{p}=0.001$ ) (Table 4).

In the analysis of amount of smoking and progression of CT findings during follow-up, 38 patients who continued smoking were divided into 18 light smokers ( $<1$ pack/day) and 20 heavy smokers ( $\geq 1$ pack/day). Progression of calcification was seen in 
Table 4. Comparison between the Patients with Progression of Calcification and Nonprogression of Calcification

\begin{tabular}{|c|c|c|c|}
\hline Variable & Progression of calcification $(\mathrm{n}=37)$ & Nonprogression of calcification $(n=22)$ & p-value \\
\hline Age, yr & $58.6 \pm 14.12$ & $60.2 \pm 14.36$ & 0.580 \\
\hline Sex, male/female & $34 / 3$ & $19 / 3$ & 0.661 \\
\hline Drinking status & & & 0.048 \\
\hline Abstinence $(\mathrm{n}=38)$ & 20 & 18 & \\
\hline Continued drinking $(\mathrm{n}=21)$ & 17 & 4 & \\
\hline Amount of alcohol consumption & & & 0.089 \\
\hline Abstinence $(\mathrm{n}=38)$ & 20 & 18 & \\
\hline Low to moderate $(<350 \mathrm{~g} / \mathrm{wk})(\mathrm{n}=14)$ & 12 & 2 & \\
\hline Heavy ( $\geq 350 \mathrm{~g} /$ wk) (n=7) & 5 & 2 & \\
\hline Status of smoking during follow-up & & & 0.001 \\
\hline No smoking* $(\mathrm{n}=21)$ & 7 & 14 & \\
\hline Continued smoking $(\mathrm{n}=38)$ & 30 & 8 & \\
\hline Amount of smoking during follow-up & & & 0.000 \\
\hline No smoking $(\mathrm{n}=21)$ & 7 & 14 & \\
\hline$<1$ pack/day $(\mathrm{n}=18)$ & 12 & 6 & \\
\hline$\geq 1$ pack/day $(\mathrm{n}=20)$ & 18 & 2 & \\
\hline
\end{tabular}

Data are presented as the mean \pm SD or number.

*Stopped smoking or maintained nonsmoking status.

Table 5. Multivariate Analysis of Alcohol Drinking and Smoking for Progression of Calcification

\begin{tabular}{lcc}
\hline & OR (95\% CI) & p-value \\
\hline Continued alcohol drinking & $1.162(0.237-5.712)$ & 0.853 \\
Amount of alcohol consumption & & 0.545 \\
Low to moderate $(<350 \mathrm{~g} / \mathrm{wk})$ & $1.624(0.223-11.814)$ & 0.632 \\
Heavy $(\geq 350 \mathrm{~g} / \mathrm{wk})$ & $0.419(0.047-3.756)$ & 0.432 \\
Continued smoking during follow-up & $9.987(1.926-51.780)$ & 0.006 \\
Amount of smoking during follow-up & & 0.008 \\
$<1$ pack/day & $6.051(1.059-34.589)$ & 0.043 \\
$\geq 1$ pack/day & $36.562(3.783-353.348)$ & 0.002 \\
\hline
\end{tabular}

OR, odds ratio; CI, confidence interval.

12 of 18 light smokers (66.7\%) and in 18 of 20 heavy smokers (90\%). Progression of calcification was associated with amount of smoking in univariate analysis ( $\mathrm{p}=0.000)$ (Table 4).

\section{Multivariate analysis of alcohol drinking and smoking for progression of calcification}

Continued alcohol drinking was not a risk factor for progression of calcification in multivariate analysis (odds ratio [OR], 1.162; 95\% confidence interval [CI], 0.237 to 5.712; $p=0.853$ ). Amount of alcohol consumption was not a risk factor of progression of calcification in multivariate analysis $(p=0.545)$. The OR in the patients with low to moderate alcohol consumption was 1.624 (95\% CI, 0.223 to $11.814 ; p=0.632$ ), and OR in those with heavy alcohol consumption was 0.419 (95\% CI, 0.047 to 3.756; $\mathrm{p}=0.437$ ) (Table 5).
Continued smoking was a risk factor of progression of calcification in chronic pancreatitis in multivariate analysis (OR, 9.987; 95\% CI, 1.926 to 51.780; $\mathrm{p}=0.006$ ). The OR for progression of calcification in the light smoker ( $<1$ pack/day) was 6.051 (95\% CI, 1.059 to $34.589 ; p=0.043$ ) and OR for progression of calcification in the heavy smoker ( $\geq 1$ pack/day) was 36.562 (95\% CI, 3.783 to $353.348 ; p=0.002$ ) in multivariate analysis. In multivariate analysis, amount of smoking was a significant predictor for progression of calcification in chronic pancreatitis $(p=0.008)$ (Table 5).

\section{Occurrence of new events during the follow-up period.}

During total of $56.3 \pm 24.5$ months of follow-up, new-onset diabetes mellitus developed in 19 patients and pancreatic cancer in one of 59 patients. 


\section{DISCUSSION}

Chronic pancreatitis is a chronic inflammatory process of the pancreas leading to destruction of exocrine tissue, fibrosis, and in some patients, loss of endocrine tissue and various clinical symptoms. ${ }^{1}$ A multiplicity of causes may lead to chronic pancreatitis. Causes of chronic pancreatitis include chronic alcohol abuse, genetic causes (e.g., mutations in the cystic fibrosis gene, hereditary pancreatitis), ductal obstruction (e.g., stone, tumor, pancreas divisum), tropical pancreatitis, systemic disease, autoimmune pancreatitis, and idiopathic pancreatitis.

The most common cause of chronic pancreatitis is chronic alcohol abuse. In developed countries, 60\% to 70\% of patients with chronic pancreatitis have a long history of heavy consumption of alcohol over 150 to $175 \mathrm{~g}$ per day before the onset of clinically apparent disease. ${ }^{8}$ Very heavy alcohol consumption is independent risk for chronic pancreatitis. In this study the most common cause was alcohol (79.7\%).

The pathogenesis of chronic pancreatitis is incompletely understood. There are four major theories to explain the pathogenesis of chronic pancreatitis: toxic-metabolic, oxidative stress, stone and duct obstruction, and necrosis-fibrosis.

Among toxic materials, alcohol was directly toxic to the acinar cell through a change in cellular metabolism. Alcohol produces cytoplasmic lipid accumulation within the acinar cells, leading to fatty generation, cellular necrosis, and eventual widespread fibrosis. ${ }^{9}$ Since less than $10 \%$ of alcoholics develop chronic pancreatitis, other predisposing factors are involved in the pathogenesis of chronic pancreatitis. ${ }^{10}$

Other toxins are linked to the development of chronic pancreatitis. For example, cigarette smoking is an independent risk factor for acute and chronic pancreatitis, and its effects could synergize with those of alcohol. ${ }^{1}$ Smoking accelerates progression of alcoholic chronic pancreatitis.

The mechanism by which smoking contributes to pancreatic injury or accelerates the pancreatic inflammatory process is still unknown. However, laboratory studies found that activation of multiple signal transduction pathways from nicotine exposure results in high levels of intracellular calcium release and may be responsible for cell cytotoxicity and cell injury in addition to pancreatic calcification. ${ }^{3,11}$

Development of calcification after initial diagnosis of chronic pancreatitis was associated with cigarette smoking. ${ }^{3}$ Pancreatic calcifications are frequent in chronic pancreatitis and considered a sign of severe or long lasting disease.

Pancreatic calcification appears to start with the intraductal formation of protein plugs, on which calcium may eventually precipitate in the form of insoluble salts, particularly calcium carbonate. Cigarette smoke contains a number of substances, all of which can exert a toxic action on pancreatic cells. Therefore smoking may have an effect in some way on the mechanisms of stone formation, thereby favoring the process of calcifica- tion. $^{12,13}$

The diagnosis of chronic pancreatitis can be made by histologic or morphologic criteria alone, or by a combination of morphologic, functional, and clinical findings. Imaging studies determining the morphologic change of pancreas are mainly used for the diagnosis recently. The imaging studies include abdominal plain films, transabdominal ultrasound, CT scan, magnetic resonance imaging, ERCP, and endoscopic ultrasound.

Abdominal CT scan is the most widely used test for the diagnosis of chronic pancreatitis and identifying the complications such as pseudocyst, abscess, necrosis, pseudoaneurysm, and bile duct stenosis. CT is useful in the evaluation of pancreas parenchyma, pancreatic duct, other organs and vessels around the pancreas. Reproducibility of CT is also high. Resolution of CT has been much improved with the introduction of multidetector $\mathrm{CT}^{14}$

CT findings of chronic pancreatitis include calcification, pancreatic duct stone, pancreatic duct dilatation and stenosis, and pancreatic parenchymal atrophy. CT has a reported sensitivity of $74 \%$ to $90 \%$ and a specificity of $85 \%$ in the diagnosis of chronic pancreatitis. ${ }^{7,15-17}$ According to a classic study, pancreatic duct dilatation was seen in $68 \%$ of patients with pancreatitis and calcification was seen in 50\%. ${ }^{15}$ Our study found that pancreatic calcification was seen in 59.3\% of patients with chronic pancreatitis.

This is a retrospective observational study. Therefore it is difficult to accurately stratify the degree of smoking and alcohol use. Attempting to obtain historical smoking data may lead to recall bias since many patients are unable to accurately report past smoking habits. Other limitations of this study are small number of included patients and relatively short duration of follow-up. Pancreatic duct dilatation was also common in CT findings of chronic pancreatitis. However, we did not include the ductal change of the pancreas in the analysis.

According to a previous study about the relationship between calcification and smoking in chronic pancreatitis with during follow-up of $10.4 \pm 6.4$ years, 376 out of 570 patients (66\%) had pancreatic calcification at the end of follow-up. Sixtyfour out of 376 patients (17\%) had pancreatic calcification at the time of diagnosis in the cumulative curve for occurrence of calcifications. ${ }^{12}$ Calcification was seen in $69 \%$ of patients with heavy smoker ( $>10$ cigarettes/day for $>10$ years) but in 55\% of patients with nonsmoker or light smoker $(<10$ cigarettes/day for $>10$ years). The relative risk of calcification in smokers versus nonsmokers was 1.21. However, there was no statistically significant correlation between alcohol and calcification. Our study showed that pancreatic calcification was 59.3\% at the time of diagnosis of pancreatitis and $88.1 \%$ at the follow-up CT scan. We studied association between progression of calcification and smoking status. Progression of calcification was seen in $78.9 \%$ of patients with smoker but in 33.3\% of patients with nonsmoker at the follow-up CT scan. In the present study, calcification of 
chronic pancreatitis progressed in continued smoking and progression of calcification was related to the amount of smoking, but the pathogenesis of toxic injury of smoking to pancreas is unknown and is needed further study.

There is a recent study about smoking status and pancreatic calcification in the patients with chronic pancreatitis who had not developed calcification. ${ }^{18}$ At the end of median 19-year follow-up, 59.8\% developed calcifications. Concerning the risk of calcifications, never-smokers and ex-smokers had similar odds ratio (0.56), but smokers of varying amount of smoking had high odds ratio (1.76 to 1.95). The authors concluded that smoking cessation seemed to reduce the risk of developing pancreatic calcification, but alcohol cessation seemed to have no influence. We found that continued cigarette smoking accelerated progression of pancreatic calcification (33.3\% in nonsmoker, $78.9 \%$ in continued smoker, $\mathrm{p}=0.006$ ) and the amount of smoking was associated with progression of calcification in patients with chronic pancreatitis $(\mathrm{p}=0.008)$ in multivariate analyses. Continued alcohol drinking accelerated pancreatic calcification in univariate analysis but not in multivariate analysis. Alcohol amount was not associated with progression of calcification in follow-up CT in the patients with chronic pancreatitis.

Many patients (29 of 50 patients) stopped alcohol drinking after the initial CT. However, only a few smokers (nine of 47 patients) stopped smoking after the initial CT. Because of this difference, there might have been no association between alcohol and pancreatic calcification. A larger and longer follow-up study is needed. The patients with chronic pancreatitis should be advised to quit smoking in the beginning of clinical onset of chronic pancreatitis.

In conclusion, we found that cigarette smoking is associated with pancreatic calcification and continued cigarette smoking accelerates progression of pancreatic calcification in patients with chronic pancreatitis.

\section{CONFLICTS OF INTEREST}

No potential conflict of interest relevant to this article was reported.

\section{REFERENCES}

1. Yadav D, Lowenfels AB. The epidemiology of pancreatitis and pancreatic cancer. Gastroenterology 2013;144:1252-1261.

2. Talamini G, Bassi C, Falconi M, et al. Alcohol and smoking as risk factors in chronic pancreatitis and pancreatic cancer. Dig Dis Sci 1999;44:1303-1311.

3. Maisonneuve P, Lowenfels AB, Müllhaupt B, et al. Cigarette smoking accelerates progression of alcoholic chronic pancreatitis. Gut 2005;54:510-514.

4. Law R, Parsi M, Lopez R, Zuccaro G, Stevens T. Cigarette smoking is independently associated with chronic pancreatitis. Pancreatology 2010;10:54-59.

5. Yadav D, Hawes RH, Brand RE, et al. Alcohol consumption, cigarette smoking, and the risk of recurrent acute and chronic pancreatitis. Arch Intern Med 2009;169:1035-1045.

6. Talamini G, Bassi C, Falconi M, et al. Cigarette smoking: an independent risk factor in alcoholic pancreatitis. Pancreas 1996;12: 131-137.

7. Hartwig W, Werner J, Ryschich E, et al. Cigarette smoke enhances ethanol-induced pancreatic injury. Pancreas 2000;21:272-278.

8. Steer ML, Waxman I, Freedman S. Chronic pancreatitis. N Engl J Med 1995;332:1482-1490.

9. Stevens T, Conwell DL, Zuccaro G. Pathogenesis of chronic pancreatitis: an evidence-based review of past theories and recent developments. Am J Gastroenterol 2004;99:2256-2270.

10. Dufour MC, Adamson MD. The epidemiology of alcohol-induced pancreatitis. Pancreas 2003;27:286-290.

11. Chowdhury P, MacLeod S, Udupa KB, Rayford PL. Pathophysiological effects of nicotine on the pancreas: an update. Exp Biol Med (Maywood) 2002;227:445-454.

12. Cavallini G, Talamini G, Vaona B, et al. Effect of alcohol and smoking on pancreatic lithogenesis in the course of chronic pancreatitis. Pancreas 1994;9:42-46.

13. Bernard JP, Adrich Z, Montalto G, et al. Inhibition of nucleation and crystal growth of calcium carbonate by human lithostathine. Gastroenterology 1992;103:1277-1284.

14. Etemad B, Whitcomb DC. Chronic pancreatitis: diagnosis, classification, and new genetic developments. Gastroenterology 2001; 120:682-707.

15. Luetmer PH, Stephens DH, Ward EM. Chronic pancreatitis: reassessment with current CT. Radiology 1989;171:353-357.

16. Remer EM, Baker ME. Imaging of chronic pancreatitis. Radiol Clin North Am 2002;40:1229-1242

17. Choueiri NE, Balci NC, Alkaade S, Burton FR. Advanced imaging of chronic pancreatitis. Curr Gastroenterol Rep 2010;12:114-120.

18. Talamini G, Bassi C, Falconi M, et al. Smoking cessation at the clinical onset of chronic pancreatitis and risk of pancreatic calcifications. Pancreas 2007;35:320-326. 